Forthcoming in Mathematical Social Sciences

\title{
Weak Pseudo-Rationalizability
}

\author{
Rush T. Stewart
}

January 8, 2020

\begin{abstract}
This paper generalizes rationalizability of a choice function by a single acyclic binary relation to rationalizability by a set of such relations. Rather than selecting those options in a menu that are maximal with respect to a single binary relation, a weakly pseudo-rationalizable choice function selects those options that are maximal with respect to at least one binary relation in a given set. I characterize the class of weakly pseudo-rationalizable choice functions in terms of simple functional properties. This result also generalizes Aizerman and Malishevski's characterization of pseudo-rationalizable choice functions, that is, choice functions rationalizable by a set of total orders.
\end{abstract}

Keywords. Aggregation; binariness; multi-criteria choice; rationalizability

\section{Introduction}

In many of the most important and influential theories of rational and social choice, preferences are assumed to be complete and transitive (e.g., Von Neumann and Morgenstern, 1944; Arrow, 1951; Savage, 1954; Anscombe and Aumann, 1963). The view that preferences are or should be so can be questioned on numerous grounds. As Sen has stressed, such a preferential structure is not required for reasoned choice. Neither incompleteness nor (certain forms of) intransitivity prevents choosing maximal alternatives, i.e., alternatives to which no other option is strictly preferred. As Sen sees it, "there is no analytical reason, nor any practical necessity, why the ranking of alternative conclusions must take this highly restrictive form" (2017, p. 455). In decision problems with finitely many options at least, acyclicity suffices for maximization. Moreover, relaxing transitivity of social preference opens up certain possibilities in the setting of social choice (e.g., Sen, 1969). There is also a sizable empirical literature on behavioral departures from transitive preferences (e.g., Tversky, 1969; Loomes et al., 1991).

Still, choosing maximal options according to an acyclic relation retains the assumption that an agent's or group's preferences are or should be given by a binary relation. It is another level of heterodoxy to relax binariness. One motivation for doing so comes from unresolved conflicts in values or goals at the time of choice. According to Sen, such unresolved conflict is the "primary source" of incompleteness (Sen, 2018, p. 11). In such cases, various values induce different preference relations on the options. There are many different decision rules one might employ in such cases. But Isaac Levi, who Sen often cites in this context (e.g., 2004; 2017, p. 460), argues that each such preference relation amounts to an evaluation of the options that the agent regards as permissible to use in choice (1986). ${ }^{1}$ So the admissible options in any menu are those that are maximal with respect to some way of evaluating the options that the agent regards as permissible. On such a view, the set of admissible options according to the set of preference relations may resist representation in terms of a single, categorical or all-things-considered binary preference relation.

\footnotetext{
${ }^{1}$ In the setting of cardinal utilities, Levi allows that an agent may not have settled on a precise weighting, i.e., a unique convex combination, of different objective functions (perhaps corresponding to primitive values or goals), even if the agent has ruled out some such weightings.
} 
In this paper, I characterize those preference structures that allow for incompleteness due to unresolved conflicts in values, while also allowing that the conflicting values themselves may fail to induce complete or transitive relations. Formally, I characterize those choice functions that, for any menu of alternatives, select those options that are maximal according to some acyclic binary relation in a set of such relations. The binary relations are not required to be weak or total orders. Such choice functions will be called weakly pseudo-rationalizable.

The are several potential interpretations, both descriptive and normative. For example, a decision maker faced with a set of options may rank them along multiple dimensions. If there is no analytic reason or practical necessity for single preference relations to take the form of weak orderings, as Sen argues, it is difficult to see why any given dimension must take such a highly restrictive form. One rule such a decision maker could follow is to restrict attention to options that are best along at least one dimension. The set of such options might also be interpreted as a type of consideration set that filters out the other available options from the decision maker's attention. Lleras et al. refer to a consideration set mapping that satisfies Sen's property $\alpha$ as a competition filter (2017). When interpreted as a consideration set map, a weakly pseudo-rationalizable correspondence is a special case of a competition filter. ${ }^{2}$

To take another possible interpretation, the set of rankings that weakly pseudo-rationalize a given choice function may already include potential ways of trading off yet more fundamental values or dimensions of comparison, but the decision maker has not settled on a uniquely acceptable form of compromise. Rather, under such unresolved conflict among candidate rankings of the options, there are various candidate rankings, corresponding to ways of trading off the fundamental values, that the decision maker has not ruled out and these form the relevant set of relations for weak pseudorationalizability. The choice-worthy options are the options that are maximal with respect to some permissible way of evaluating the options, some way the decision maker hasn't ruled out. This interpretation extends Levi's philosophical motivation for $E$-admissibility beyond the expected utility setting. Levi has long advocated a theory that allows both probabilities and utilities to be "indeterminate" (1974). Given a set of probabilities $\mathbb{P}$ over states and a set of utilities $\mathbb{U}$ over consequences of options, an option $x$ is said to be $E$-admissible in a menu of alternatives $Y$ if there is some probability utility pair $(p, u)$ with $p \in \mathbb{P}$ and $u \in \mathbb{U}$ such that $x$ maximizes $p$-expected $u$-utility in $Y$. In such a framework, the probability-utility pairs induce various weak order rankings of the options and Levi's $E$-admissibility rule selects those options in a menu that are maximal according to some ranking. These ideas have been extended in various ways by Seidenfeld, Schervish, and Kadane (e.g., 1995; 2010). Choice functions generated by Levi's $E$-admissibility criterion generally violate binariness. But they do nonetheless enjoy a certain discernible structure. Rather than being rationalizable by a single complete and transitive relation, they are pseudo-rationalizable by a set of such relations. Independently of Levi's work, Aizerman and Malishevski characterize pseudo-rationalizable choice functions in terms of simple properties of abstract choice functions (Aizerman and Malishevski, 1981). This work (and more) is reviewed in (Aizerman, 1985) and extended in (Aleskerov et al., 2007), but there does not appear to be a characterization of the more general case in which the set of relations used to rationalize a choice function consists of merely acyclic relations. Given that standard presentations of choice theory typically distinguish rationalizability of a choice function (by an acyclic binary relation) from stronger concepts like rationalizability by particular types of orderings, Theorem 4 fills an obvious gap in the literature extending rationalizability concepts to rationalizability by sets of relations.

Other studies explore using multiple relations or "rationales" to explain choice behavior. Kalai

\footnotetext{
${ }^{2}$ Relatedly, Duggan studies a weak notion of rationalizability that he labels "proto-rationalizability." Protorationalizable choice functions can be represented as taking a certain union of intersections of the maximal elements according to a set of acyclic relations and are characterized by Sen's property $\alpha$ (Duggan, 2019, Theorem 6). With the help of an example, Duggan observes that removing the intersections is not possible: "Theorem 6 is not true if proto-rationalizability is stated with only a union (rather than a union of intersections) of acyclic relations" (2019, p. 22). In Duggan's terminology, our main result, Theorem 4 below, provides a characterization of the version of proto-rationalizability stated with only a union of acyclic relations.
} 
et al. (2002) work in the framework of element-valued choice functions and introduce the notion of rationalization by multiple rationales. An element-valued choice function is said to be rationalized by a set of total orderings if the chosen option from a menu is maximal with respect to some ordering in the set. The central notion of this paper, weak pseudo-rationalizability, is one way to generalize rationalization by multiple rationales to set-valued choice functions while relaxing the requirement that the rationales be total orders. So, we make less restrictive assumptions about the nature of rationales. Manzini and Mariotti's sequentially rationalizable choice also appeals to a fixed set of rationales to rationalize an element-valued choice function. However, they do not require that the rationales be total orders and, importantly, the rationales are applied sequentially to eliminate inferior options (2007). Manzini and Mariotti interpret sequentially rationalizable choice as a form of bounded rationality, one that allows for some but not all departures from "the standard model of rationality." A similar interpretation is possible for weakly pseudo-rationalizable choice functions. If, unlike Sen or Levi, one thinks of rational (categorical) preferences as inducing a weak ordering of the options (or at least inducing a binary preference relation rationalizing choice), weak pseudo-rationalizability could be conceived of as choice behavior that violates some but not all rationality constraints. Our characterization of weakly pseudo-rationalizable choice functions facilitates comparisons to characterizations of rationalizability (Theorem 1) and rationalizability by a weak ordering (Theorem 2) so that possible departures from the standard model of rationality are apparent. Yet another interpretation of weakly pseudo-rationalizable choice, then, is as a certain form of boundedly rational choice.

After rehearsing the basic notions relating to binary relations, choice functions, and rationalizability that will be employed in Section 2, I briefly review subrationalizability and pseudo-rationalizability in Section 3. In Section 4, I introduce an axiom - a weakening of standard expansion consistencythat, along with Sen's $\alpha$, allows us to state a characterization of weak pseudo-rationalizability (Theorem 4).

\section{Preliminaries}

Let $X$ be a finite set of options. A preference relation on $X$ is a binary relation $R \subseteq X \times X$. We will use the infix and graph notations for $R, x R y$ and $(x, y) \in R$, interchangeably. We call $R$ asymmetric if $x R y$ implies $\neg y R x$. Any binary relation $R$ can be decomposed into its asymmetric and symmetric parts, $P$ and $I$, respectively, by putting $x P y$ if and only if $x R y$ and $\neg y R x$ and $x I y$ if and only if $x R y$ and $y R x$. Clearly, $R=P \cup I$. If we think of $R$ as a preference relation, then $P$ represents strict preference and $I$ represents indifference. If $x R y$ and $y R z$ implies $x R z, R$ is called transitive. We say that $R$ is acyclic if, for any $k \in \mathbb{N}, x_{1} P x_{2}, x_{2} P x_{3}, \ldots, x_{k-1} P x_{k}$ implies $\neg x_{k} P x_{1}$. If $x R y$ or $y R x$ for all $x, y \in X, R$ is complete. A relation that is complete and transitive is a weak order. If $R$ is also antisymmetric - $x R y$ and $y R x$ implies $x=y$-then it is called a total order.

Define $\mathcal{X}$ to be the set of all non-empty subsets of $X$. We will refer to elements of $\mathcal{X}$ as menus. A choice function $C: \mathcal{X} \rightarrow \mathcal{X}$ associates a choice set $C(Y) \subseteq Y$ to every menu $Y \in \mathcal{X}$. Note that we require $C(Y) \neq \emptyset$. A binary relation $R$ generates a choice function $C_{R}$ by determining the maximal elements on any given menu, $M(Y, R)$.

$$
\forall Y \in \mathcal{X}, C_{R}(Y)=\{x \in Y: y P x \text { for no } y \in Y\}=M(Y, R)
$$

Starting with a choice function, when can it be regarded as selecting the maximal elements according to some binary relation for any menu? If there exists a relation $R$ such that

$$
\forall Y \in \mathcal{X}, C(Y)=\{x \in Y: y P x \text { for no } y \in Y\}
$$

then $C$ is said to be rationalizable. Since we are considering choice functions on the unrestricted domain of non-empty subsets of $X$, only acyclic relations can rationalize choice functions. If $R$ contains a cycle $x_{1} P x_{2}, x_{2} P x_{3}, \ldots, x_{k-1} P x_{k}, x_{k} P x_{1}$, then $C\left(\left\{x_{i}: i=1, . ., k\right\}\right)=\emptyset$, contradicting the fact that $C$ is a choice function. For selecting maximal elements, $R$ need not be complete. However, maximizing 
an incomplete but acyclic relation $R$ can be mimicked by optimizing a complete relation $R^{\prime}$, where $x R^{\prime} y$ if and only if $\neg y P x$. Essentially, $R^{\prime}$ converts any incompleteness of $R$ into indifference. Now we have $x \in C_{R}(Y)=M(Y, R)$ if and only if $x \in C_{R^{\prime}}(Y)=\{x \in Y: x R y$ for all $y \in Y\}$. Sen has made much of the distinction between maximization and optimization (e.g., Sen, 1997, 2017, chs. 1*, A6).

There are a number of ways to think of a choice function as revealing an underlying preference relation. For example, we could say $x R_{s} y$ if $x \in C(Y)$ for some menu such that $y \in Y$. That is, $x$ is selected in the presence of $y$. Or we could put $x R_{b} y$ if $x \in C(\{x, y\})$. This is typically called the base relation for $C$. Unlike the base relation, $R_{s}$ is always acyclic. The two relations coincide if the choice function satisfies perhaps the most central axiomatic constraint on choice functions, so-called contraction consistency also known as Chernoff and Sen's property $\alpha$.

$(\alpha) \quad$ If $S \subseteq T$, then $S \cap C(T) \subseteq C(S)$

Proposition 1. Let $C$ be a choice function on $X$. If $C$ satisfies property $\alpha$, then $R_{b}=R_{s}$.

Another central constraint in the theory of choice is often dubbed expansion consistency or Sen's property $\gamma$. Since we are assuming that $X$ is finite, we can express it in the following simple form.

$(\gamma) \quad C(S) \cap C(T) \subseteq C(S \cup T)$

Together, contraction and expansion consistency are equivalent to rationalizability.

Theorem 1. (e.g., Sen, 1971, Theorem 9) A choice function $C: \mathcal{X} \rightarrow \mathcal{X}$ is rationalizable if and only if it satisfies properties $\alpha$ and $\gamma$.

It is a straightforward exercise to verify that $C$ is rationalizable if and only if it is rationalizable by its base relation. Rationalizability by certain types of relations may be of particular interest. For instance, when can $C$ be regarded as selecting the maximal elements according to some ranking or weak order? Sen's property $\beta$ does not imply $\gamma$ in general, but in the presence of $\alpha$, it does.

( $\beta) \quad$ If $S \subseteq T, x, y \in C(S)$, and $x \in C(T)$, then $y \in C(T)$

Properties $\alpha$ and $\beta$ characterize rationalizability by a weak order.

Theorem 2. (e.g., Sen, 1977, Proposition 11) A choice function $C: \mathcal{X} \rightarrow \mathcal{X}$ is rationalizable by a weak order if and only if it satisfies properties $\alpha$ and $\beta$.

Since Sen's work, presentations of choice theory standardly distinguish rationalizability from the stronger notion of rationalizability by a weak order, just as we have here. Yet, in generalizing to rationalizability by sets of binary relations, there is an analogue of Theorem 2, but, as far as I know, no analogue of Theorem 1 . Theorem 4 below fills this lacuna in the literature.

\section{Pseudo-Rationalizability}

The development in this section closely follows Moulin $(1985, \S 5)$. A choice function is called subrationalizable if it contains a rationalizable choice function. Equivalently, for some acyclic relation $R$,

$$
M(Y, R) \subseteq C(Y) \text {, for all } Y \in \mathcal{X} .
$$

Moulin reports that this property was introduced by Deb (1983) under the name weak rationalizability (1985, p. 155). That seems to be not quite correct, as Deb refers to this property as weak representability (1983, p. 100). For finite sets, subrationalizability is equivalent to subrationalizability by a total order. 
Lemma 1. (Moulin, 1985, Lemma 4) A choice function $C: \mathcal{X} \rightarrow \mathcal{X}$ is subrationalizable if and only if there is a strict total order $R$ such that $M(Y, R) \subseteq C(Y)$ for all $Y \in \mathcal{X}$.

Since $R$ is a total order, it has a unique maximum in every menu, so $C_{R}(Y)$ will be a singleton for every $Y \in \mathcal{X}$. One point worth emphasizing for our purposes is the distinction between a choice function's being subrationalizable and its being subrationalizable in a way that, for a particular option $x \in C(S)$ and menu $S$, delivers $x \in C_{R}(S)$. It does not follow from Lemma 1 that if $C$ is subrationalizable and $x \in C(S)$, then there is a total order $R$ such that $C_{R}(S)=\{x\}$ and $C_{R}(Y) \subseteq C(Y)$ for all $Y \in \mathcal{X}$. (Consider $C_{1}$ in Example 1 with $y \in C_{1}(\{x, y\})$.)

Lemma 2. (Moulin, 1985, Lemma 5) Let $C$ be a choice function on $X$. If $C$ satisfies property $\alpha$, then $C$ is subrationalizable.

When is it the case that a choice function's total order subrationalizations generate the choice function? Let $N$ be a finite set of total orders of $X,\left\{R_{i}: i=1, \ldots, n\right\}$. Each $R_{i}$ generates a totalorder rationalizable choice function, $C_{i}$. We say that $C$ is pseudo-rationalizable if there exists such a set of total orders such that

$$
\forall Y \in \mathcal{X}, C(Y)=\bigcup_{i \in N} C_{i}(Y)
$$

Aizerman and Malishevski also call this collected extremal choice. Pseudo-rationalizability can be characterized in terms of $\alpha$ and the following axiom often referred to as Aizerman's axiom.

(Aiz) If $C(T) \subseteq S \subseteq T$, then $C(S) \subseteq C(T)$

Theorem 3. (Aizerman and Malishevski, 1981, Theorem 3; Moulin, 1985, Theorem 5) A choice function $C$ on $X$ is pseudo-rationalizable iff it satisfies $\alpha$ and Aiz. ${ }^{3}$

If $C$ is pseudo-rationalizable by a set of total orders, it is clearly rationalizable by a set of weak orders: total orders are weak orders. The converse is also true. One way to see this is that properties $\alpha$ and $\beta$, the properties characterizing rationalizability by a weak order, jointly imply Aizerman's axiom. So any weak order rationalizable choice function is pseudo-rationalizable by a set of total orders. Thus, if $C$ is pseudo-rationalizable by a set of weak order rationalizable choice functions, it is pseudo-rationalizable by a (generally larger) set of total order rationalizable choice functions.

Pseudo-rationalizability (Theorem 3) generalizes rationalizablity by a weak order (Theorem 2) to rationalizability to a set of weak orders. It is natural, then, to inquire into the analogous generalization of rationalizability tout court (Theorem 1) to rationalizability by a set of acyclic binary relations. We turn to this issue now.

\section{Weak Pseudo-Rationalizability}

What if we relax the assumption that the subrationalizations must be total (or weak) orders? Now we are concerned only that there exists a finite set $N$ of binary relations on $X,\left\{R_{i}: i=1, \ldots, n\right\}$, each of which generates a rationalizable choice function, $C_{i}(Y)=M\left(Y, R_{i}\right)=\left\{x \in Y: y P_{i} x\right.$ for no $\left.y \in Y\right\}$, that pseudo-rationalizes a choice function, $C$. Weakly pseudo-rationalizable choice functions, put another way, are those for which there exists a finite set of rationalizable choice functions, $\left\{C_{i}: i=\right.$ $1, \ldots, n\}$, such that

\footnotetext{
${ }^{3}$ Pseudo-rationalizability can also be characterized in terms of a single axiom due to Plott known as path independence: for all $S, T \in \mathcal{X}, C(S \cup T)=C(C(S) \cup C(T))$. Choice functions satisfying path independence, i.e., pseudorationalizable choice functions, have been used to study matching markets (e.g., Chambers and Yenmez, 2017).
} 


$$
\forall Y \in \mathcal{X}, C(Y)=\bigcup_{i \in N} C_{i}(Y) .
$$

Again, we do not require that the choice functions $C_{i}$ be rationalizable by a weak or total order, only by some (acyclic) binary relation.

Weak pseudo-rationalizability implies neither expansion consistency nor Aizerman's axiom as the following example illustrates.

Example 1. Let $X=\{x, y, z\}$. Consider two rationalizable choice functions, $C_{1}, C_{2}$, and $C$ such that $C(S)=C_{1}(S) \cup C_{2}(S)$. Choice sets for singletons are defined in the obvious way.

$$
\begin{aligned}
& C_{1}(X)=\{x\} \\
& C_{2}(X)=\{x\} \\
& C_{1}(\{x, y\})=\{x, y\} \\
& C_{2}(\{x, y\})=\{x\} \\
& C_{1}(\{x, z\})=\{x\} \\
& C_{2}(\{x, z\})=\{x, z\} \\
& C_{1}(\{y, z\})=\{z\} \\
& C_{2}(\{y, z\})=\{y\} \\
& C(X)=\{x\} \\
& C(\{x, y\})=\{x, y\} \\
& C(\{x, z\})=\{x, z\} \\
& C(\{y, z\})=\{y, z\}
\end{aligned}
$$

To see that $C$ violates property $\gamma$, notice, for example, that $y \in C(\{x, y\}) \cap C(\{y, z\})$, but $y \notin C(X)$. For a violation of Aizerman's axiom, observe that $\{x, y\} \subseteq X$ and $C(X) \subseteq\{x, y\}$, but $C(\{x, y\}) \nsubseteq$ $C(X)$.

The following example illustrates that not all choice functions satisfying contraction consistency are weakly pseudo-rationalizable.

Example 2. Let $X=\{x, y, z\}$ and consider the following choice function on $X$. Again, choice sets for singletons are defined in the obvious way.

$$
\begin{aligned}
C(X) & =\{x\} \\
C(\{x, y\}) & =\{x, y\} \\
C(\{x, z\}) & =\{x, z\} \\
C(\{y, z\}) & =\{y\}
\end{aligned}
$$

It is easy to check that $C$ satisfies property $\alpha$. It does not satisfy property $\gamma$ since $y \in C(\{x, y\} \cap$ $C(\{y, z\})$ but $y \notin C(X)$. If $C$ were weakly pseudo-rationalizable, then, for each $i \in N, C_{i}(\{y, z\})=$ $\{y\}$. But for some $j \in N$, we must have $y \in C_{j}(\{x, y\})$. Since it is assumed that $C_{j}$ is rationalizable, by property $\gamma, y \in C_{j}(X)$, in turn implying that $y \in C(X)$, contrary to the definition of $C$.

So weak pseudo-rationalizability is not a trivial property in the presence of property $\alpha$.

One idea is simply to restrict the sorts of violations of expansion consistency that a choice function can exhibit. The following property, which we call weak $\gamma$, blocks the pattern displayed in Example 2 and is generally satisfied by weakly pseudo-rationalizable choice functions. It clearly weakens property $\gamma$ while placing limits on the ways in which the axiom can be violated.

(Weak $\gamma$ ) If $C(S)=\{x\}$ and $x \in C(T)$, then $x \in C(S \cup T)$

In terms of the representation we are seeking which appeals to a set of relations, the reason that property $\gamma$ can fail is that an option might be maximal according to $R$ in $S$ and maximal according to $R^{\prime}$ in $T$, but not maximal according to either (or any) in $S \cup T$ (as is the case for $y$ in Example 1). This situation is not possible when an option is uniquely admissible in $S$ or $T$ (as is the case for $y$ in Example 2 ). Put informally, the axiom says that if an option is best according to every rationale in one menu, 
and best according to some rationale in another menu, then the option must be best according to some rationale in the union of the two menus. It is notable that this key axiom refers centrally to unique admissibility. Moreover, while properties $\alpha$ and $\gamma$ characterize rationalizability by a binary relation, $\alpha$ and weak $\gamma$ - the latter an exceedingly simple modification of $\gamma$-jointly characterize rationalizability by a set of binary relations. Perhaps greater insight could be achieved eventually by finding a more handsome but equivalent (in the presence of $\alpha$ at least) axiom. From a normative point of view, though, weak $\gamma$ must be regarded as at least as compelling as property $\gamma$ which implies it.

Weak $\gamma$ also constrains the sorts of failures of Aizerman's axiom that $C$ can display.

Proposition 2. Let $C$ be a choice function on $X$ satisfying weak $\gamma$. If $C(T) \subseteq S \subseteq T$ but $C(S) \nsubseteq$ $C(T)$, then for any $x \in C(S) \backslash C(T), C(T \backslash C(T)) \neq\{x\}$.

Proof. Suppose $C(T) \subseteq S \subseteq T$, and let $x \in C(S) \backslash C(T)$. If $x \in C(T \backslash C(T))$, then, since $x \in$ $C(S) \cap C(T \backslash C(T))$ but $x \notin C(S \cup T \backslash C(T))=C(T)$, weak $\gamma$ implies that $C(S) \neq\{x\} \neq C(T \backslash C(T))$. If $x \notin C(T \backslash C(T))$, clearly $\{x\} \neq C(T \backslash C(T))$.

Together, property $\alpha$ and Aizerman's axiom imply weak $\gamma$.

Proposition 3. Let $C$ be a choice function on $X$. If $C$ satisfies properties $\alpha$ and Aiz, then $C$ satisfies weak $\gamma$.

Proof. Let $C$ satisfy $\alpha$ and Aiz. Suppose that $C(S)=\{x\}$ and that $x \in C(T)$. We want to show that $x \in C(S \cup T)$. For any $y \neq x$ such that $y \in C(S \cup T), y \in S \cup T$, so $\alpha$ implies that $y \in C(S)$ or $y \in C(T)$. By our assumption that $C(S)=\{x\}$, we must have $y \in C(T)$. Hence, $C(S \cup T) \subseteq T \subseteq S \cup T$. By Aiz, it follows that $C(T) \subseteq C(S \cup T)$. Since $x \in C(T)$, we have $x \in C(S \cup T)$ as desired.

However, properties $\alpha$ and weak $\gamma$ do not imply Aizerman's axiom, as Example 1 demonstrates. Moreover, properties $\alpha$ and Aiz do not imply standard expansion consistency $\gamma \cdot{ }^{4}$ In light of our main result below, Proposition 3 is not surprising. Along with property $\alpha$, weak $\gamma$ allows us to state a simple characterization of weak pseudo-rationalizability. So, if $C$ is pseudo-rationalizable by a set of total orders, thus satisfying properties $\alpha$ and Aiz, then $C$ is weakly pseudo-rationalizable by a set of acyclic relations, thus satisfying properties $\alpha$ and weak $\gamma$.

Before proving the main result, we introduce one more concept that plays a central role in the proof. Define the set of options that $x$ weakly pseudo-dominates as follows.

$$
D_{x}:=\{y \in X \backslash\{x\}: \exists Y \in \mathcal{X} \text { such that } y \in Y \text { and } C(Y)=\{x\}\}
$$

As the categorical choice function, $C$, in Example 1 shows, $y \in D_{x}$ does not imply that $y$ is never chosen from a menu containing $x$. Property $\alpha$ allows us to state more about $D_{x}$.

Lemma 3. Let $C$ be a choice function on $X$ and $x \in X$. If $C$ satisfies property $\alpha$, then

(i) $C\left(D_{x} \cup\{x\}\right)=\{x\}$, and

(ii) $y \in D_{x}$ implies $x \notin D_{y}$.

Proof. Let $C$ be a choice function on $X$ that satisfies property $\alpha$ and suppose $x \in X$.

(i). To see that $C\left(D_{x} \cup\{x\}\right)=\{x\}$, observe that if $C(U)=\{x\}$, then $U \subseteq D_{x} \cup\{x\}$. Suppose there is a $z \neq x$ such that $z \in C\left(D_{x} \cup\{x\}\right)$. Since $z \in D_{x}$, then, there is some $U_{z} \subseteq D_{x} \cup\{x\}$ such that $z \in U_{z}$ and $C\left(U_{z}\right)=\{x\}$. By $\alpha, z \in C\left(U_{z}\right)$, which is a contradiction. Hence, $(i)$.

(ii). Let $y \in D_{x}$ and suppose $x \in D_{y}$. By assumption, there exist menus $U_{x}, U_{y} \in \mathcal{X}$ such that $x \in U_{x}$ and $C\left(U_{x}\right)=\{y\}$ and $y \in U_{y}$ and $C\left(U_{y}\right)=\{x\}$. Since $U_{x} \subseteq U_{x} \cup U_{y}$, by $\alpha, U_{x} \cap C\left(U_{x} \cup U_{y}\right) \subseteq$ $C\left(U_{x}\right)=\{y\}$. So, $x \notin C\left(U_{x} \cup U_{y}\right)$. Analogously, $\alpha$ implies $U_{y} \cap C\left(U_{x} \cup U_{y}\right) \subseteq C\left(U_{y}\right)=\{x\}$. It follows that $C\left(U_{x} \cup U_{y}\right)=\emptyset$, contradicting the assumption that $C$ is a choice function. Hence, $($ ii $) .{ }^{5}$

\footnotetext{
${ }^{4}$ Consider $X=\{x, y, z\}$ and two total orderings $R_{1}$ and $R_{2}$ with $x R_{1} y, y R_{1} z$ and $z R_{2} y, y R_{2} x$. Letting $C$ denote the pseudo-rationalzable choice function generated from $R_{1}$ and $R_{2}$, we have $y \in C(\{x, y\}) \cap C(\{y, z\})$, but $y \notin C(X)$.

${ }^{5}$ Thanks to an anonymous referee for suggesting a shorter proof Lemma 3.ii.
} 
We conclude with the statement and proof of the main result.

Theorem 4. A choice function $C: \mathcal{X} \rightarrow \mathcal{X}$ is weakly pseudo-rationalizable if and only if it satisfies $\alpha$ and weak $\gamma$.

Proof. $(\Rightarrow)$. Let $C$ be weakly pseudo-rationalizable by the collection $\left\{C_{i}: i \in N\right\}$ of rationalizable choice functions: for all $S \in \mathcal{X}, C(S)=\bigcup_{i \in N} C_{i}(S)$. We show that $C$ satisfies $\alpha$ and weak $\gamma$ in turn. Let $S \subseteq T$ and suppose $x \in S \cap C(T)$. So, $x \in S$ and, for some $i \in N, x \in C_{i}(T)$. Since $C_{i}$ satisfies property $\alpha, x \in C_{i}(\{x, y\})$ for all $y \in T$. Since $S \subseteq T$, we have $x \in C_{i}(\{x, y\})$ for all $y \in S$. By property $\gamma$, it follows that $x \in C_{i}(S)$. Hence, $x \in C(S)$ and $C$ satisfies property $\alpha$.

Now, weak $\gamma$. Suppose that $C(S)=\{x\}$ and that $x \in C(T)$. We want to show that $x \in C(S \cup T)$. Since $C(S)=\{x\}$, it follows that for all $i \in N, C_{i}(S)=\{x\}$. Since $x \in C(T)$, it follows that, for some $j \in N, x \in C_{j}(T)$. Hence, $x \in C_{j}(S) \cap C_{j}(T)$, and since $C_{j}$ satisfies property $\gamma, x \in C_{j}(S \cup T)$. This, in turn, implies that $x \in C(S \cup T)$ as desired. So, $C$ satisfies weak $\gamma$.

$(\Leftarrow)$. Suppose that $C$ satisfies properties $\alpha$ and weak $\gamma$. There are finitely many acyclic subrationalizations, $R_{1}, \ldots, R_{n}$, of $C$. We need to prove that $C(Y)$ is a subset of $\bigcup_{i \in N} C_{i}(Y)$ for any $Y$, where $C_{i}(Y)=M\left(Y, R_{i}\right)$. (The other inclusion is trivial since each acyclic subrationalization $R$ generates a choice function $C_{R}$ such that $C_{R}(Y) \subseteq C(Y)$ for any menu $Y$.) Let $S \subseteq X$ and $x \in C(S)$. We must find an acyclic relation $R$ satisfying

$$
x \in M(S, R)
$$

and

$$
\forall Y \in \mathcal{X}, M(Y, R) \subseteq C(Y)
$$

Recall the set of options that $x$ weakly pseudo-dominates from (2).

$$
D_{x}=\{y \in X \backslash\{x\}: \exists Y \in \mathcal{X} \text { such that } y \in Y \text { and } C(Y)=\{x\}\}
$$

We will use $D_{x}$ to define a choice function $C^{\prime}$ such that $C^{\prime}(Y) \subseteq C(Y)$ for all $Y \in \mathcal{X}$. Then, we will prove that $C^{\prime}$ satisfies $\alpha$ so that we can appeal to Lemma 2 for the existence of a subrationalizing total order. This total order may lack property (3). So we define a new, acyclic but incomplete relation on the basis of the total order that is guaranteed to satisfy (3) and prove that it preserves property (4).

Define the choice function $C^{\prime}$ by

$$
C^{\prime}(Y):=\left\{\begin{array}{l}
C(Y) \backslash\{x\}, \text { if } Y \backslash\left(D_{x} \cup\{x\}\right) \neq \emptyset ; \\
C(Y), \text { otherwise. }
\end{array}\right.
$$

Observe that $C^{\prime}$ is a well-defined choice function. By construction we have $C^{\prime}(Y) \subseteq C(Y) \subseteq Y$ for all $Y \in \mathcal{X}$. To see that $C^{\prime}(Y) \neq \emptyset$, notice that if $C(Y)=\{x\}$, then $Y \backslash\left(D_{x} \cup\{x\}\right)=\emptyset$. So $C^{\prime}(Y)=C(Y) \neq \emptyset$.

We now show that $C^{\prime}$ satisfies property $\alpha$. Let $Y \subseteq U \subseteq X$ and $w \in C^{\prime}(U) \cap Y$. We want to show that $w \in C^{\prime}(Y)$. If $w=x$, then $U \backslash\left(D_{x} \cup\{x\}\right)=\emptyset$ and $C^{\prime}(U)=C(U)$ by the definition of $C^{\prime}$. Since $C$ satisfies property $\alpha, w \in C(Y)$. And since $Y \subseteq U$, we have $Y \backslash\left(D_{x} \cup\{x\}\right)=\emptyset$. So, $C^{\prime}(Y)=C(Y)$. It follows that $w \in C^{\prime}(Y)$ as desired. If $w \neq x$, then, since $C^{\prime}(Y)=C(Y)$ or $C^{\prime}(Y)=C(Y) \backslash\{x\}$, $w \in C^{\prime}(Y)$. This establishes that $C^{\prime}$ satisfies property $\alpha$.

By $\alpha$ and Lemma 2, there is a subrationalizing total order preference relation $R$ such that

$$
\forall Y \in \mathcal{X}, M(Y, R) \subseteq C^{\prime}(Y) .
$$

In general, there are several such relations. Obviously, if $M(S, R)=\{x\}$ for some such $R$, we are done. So suppose that for no subrationalizing strict preference relation $R$ do we have $M(S, R)=\{x\}$. Pick a total order subrationalizing relation $R$ and define

$$
R^{\prime}:=R \backslash\{(y, x): y \in S \backslash\{x\}\} .
$$


Since the relation $R$ is acyclic, $R^{\prime}$ is as well-though $R^{\prime}$ is no longer complete - since we introduced no new strict preferences. ${ }^{6}$ Observe that we have $y R^{\prime} x$ for no $y \in S$ so $y P^{\prime} x$ for no $y \in S$. Thus, $x \in M\left(S, R^{\prime}\right)$, thereby securing $(3){ }^{7}$

Finally, we need to show that $M\left(\cdot, R^{\prime}\right)$ satisfies (4). Notice that if $x \notin M\left(Y, R^{\prime}\right)$, we are done: $M\left(Y, R^{\prime}\right)=C_{R}(Y) \subseteq C^{\prime}(Y) \subseteq C(Y)$. So suppose that $x \in M\left(Y, R^{\prime}\right)$. For any $y \notin D_{x} \cup\{x\}$, $C^{\prime}(\{x, y\})=\{y\}$ by construction. Hence, $y P x$. If, in addition, $y \notin S$, we have $y P^{\prime} x$. So, it must be the case that $Y \subseteq S \cup\left(D_{x} \cup\{x\}\right)=S \cup D_{x}$ since $x \in M\left(Y, R^{\prime}\right)$. We have $x \in C(S)$ by assumption. Since $C$ satisfies property $\alpha$, by Lemma 3.i, $C\left(D_{x} \cup\{x\}\right)=\{x\}$. Weak $\gamma$ now implies that $x \in C\left(S \cup\left(D_{x} \cup\{x\}\right)\right)=C\left(S \cup D_{x}\right)$ since $x \in C(S)$ and $C\left(D_{x} \cup\{x\}\right)=\{x\}$. By $\alpha, x \in C(Y)$ since, as we have just shown, $Y \subseteq S \cup\left(D_{x} \cup\{x\}\right)$. For any other $y \in M\left(Y, R^{\prime}\right)$, we must have $y \in C_{R}(Y) \subseteq C^{\prime}(Y) \subseteq C(Y)$ because $R^{\prime}$ modifies $R$ only by removing any strict preferences for options in $S$ over $x$. Hence, $M\left(Y, R^{\prime}\right) \subseteq C(Y)$ for all $Y \in \mathcal{X}$. We have shown that if $C$ satisfies properties $\alpha$ and weak $\gamma$, then $C$ is weakly pseudo-rationalizable.

\section{References}

Aizerman, M. and A. Malishevski (1981). General theory of best variants choice: Some aspects. Automatic Control, IEEE Transactions on 26(5), 1030-1040.

Aizerman, M. A. (1985). New problems in the general choice theory. Social Choice and Welfare 2(4), $235-282$.

Aleskerov, F., D. Bouyssou, and B. Monjardet (2007). Utility Maximization, Choice and Preference, Volume 16. Springer Science \& Business Media.

Anscombe, F. J. and R. J. Aumann (1963). A definition of subjective probability. The Annals of Mathematical Statistics 34(1), 199-205.

Arrow, K. J. (2012, originally published in 1951). Social Choice and Individual Values. Martino Publishing.

Chambers, C. P. and M. B. Yenmez (2017). Choice and matching. American Economic Journal: Microeconomics 9(3), 126-147.

Deb, R. (1983). Binariness and rational choice. Mathematical Social Sciences 5(1), 97-105.

Duggan, J. (2019). Weak rationalizability and Arrovian impossibility theorems for responsive social choice. Public Choice 179(1-2), 7-40.

Kalai, G., A. Rubinstein, and R. Spiegler (2002). Rationalizing choice functions by multiple rationales. Econometrica 70(6), 2481-2488.

Levi, I. (1974). On indeterminate probabilities. The Journal of Philosophy 71(13), 391-418.

Levi, I. (1986). Hard choices: Decision making under unresolved conflict. Cambridge University Press.

Lleras, J. S., Y. Masatlioglu, D. Nakajima, and E. Y. Ozbay (2017). When more is less: Limited consideration. Journal of Economic Theory 170, 70-85.

Loomes, G., C. Starmer, and R. Sugden (1991). Observing violations of transitivity by experimental methods. Econometrica: Journal of the Econometric Society 59(2), 425-439.

Manzini, P. and M. Mariotti (2007). Sequentially rationalizable choice. American Economic Review 97(5), $1824-1839$.

\footnotetext{
${ }^{6}$ Alternatively, we could have defined $R^{\prime}$ by $R \cup\{(x, y): y \in S\}$ which yields an acyclic relation with a strict part that is not necessarily transitive. The remainder of the argument goes through either way: $x \in M\left(S, R^{\prime}\right)$ and $M\left(Y, R^{\prime}\right) \subseteq C(Y)$ for all $Y \in \mathcal{X}$.

${ }^{7}$ Benedikt Höltgen, who at the time of writing is an MA student and TA for my introductory decision theory course at the MCMP, pointed out an alternative proof involving an elegant inductive construction of a strict partial order that can be modified the same way that we have modified $R$ to complete the proof.
} 
Moulin, H. (1985). Choice functions over a finite set: a summary. Social Choice and Welfare 2(2), 147-160.

Savage, L. (1972, originally published in 1954). The Foundations of Statistics. New York: John Wiley and Sons.

Seidenfeld, T., M. J. Schervish, and J. B. Kadane (1995). A representation of partially ordered preferences. The Annals of Statistics 23(6), 2168-2217.

Seidenfeld, T., M. J. Schervish, and J. B. Kadane (2010). Coherent choice functions under uncertainty. Synthese 172(1), 157-176.

Sen, A. (1969). Quasi-transitivity, rational choice and collective decisions. The Review of Economic Studies 36(3), 381-393.

Sen, A. (1971). Choice functions and revealed preference. The Review of Economic Studies 38(3), 307-317.

Sen, A. (1977). Social choice theory: A re-examination. Econometrica: Journal of the Econometric Society $45(1), 53-88$.

Sen, A. (1997). Maximization and the act of choice. Econometrica: Journal of the Econometric Society 65(4), 745-779.

Sen, A. (2004). Incompleteness and reasoned choice. Synthese 140(1), 43-59.

Sen, A. (2017). Collective Choice and Social Welfare (Expanded Edition). Penguin.

Sen, A. (2018). The importance of incompleteness. International Journal of Economic Theory 14(1), 9-20.

Tversky, A. (1969). Intransitivity of preferences. Psychological Review 76(1), 31-48.

Von Neumann, J. and O. Morgenstern (2007, originally published in 1944). Theory of Games and Economic Behavior (Commemorative Edition). Princeton University Press. 